\title{
Effect of parecoxib combined with thoracic epidural analgesia on pain after thoracotomy
}

\author{
Xiao-Min Ling*, Fang Fang*, Xiao-Guang Zhang, Ming Ding, Qiu-A-Xue Liu, Jing Cang \\ Department of Anesthesiology, Zhongshan Hospital, Fudan University, Shanghai 200032, China \\ Contributions: (I) Conception and design: XM Ling, F Fang, XG Zhang, J Cang; (II) Administrative support: J Cang; (III) Provision of study materials \\ or patients: All authors; (IV) Collection and assembly of data: XM Ling, F Fang; (V) Data analysis and interpretation: XM Ling, F Fang, XG Zhang; \\ (VI) Manuscript writing: All authors; (VII) Final approval of manuscript: All authors. \\ *These authors contributed equally to this work. \\ Correspondence to: Jing Cang. Department of Anaesthesiology, Zhongshan Hospital, Fudan University, 180 Fenglin Rd, Shanghai 200032, China. \\ Email: cangjing_zs@sina.com.
}

\begin{abstract}
Background: Thoracotomy results in severe postoperative pain potentially leading to chronic pain. We investigated the potential benefits of intravenous parecoxib on postoperative analgesia combined with thoracic epidural analgesia (TEA).
\end{abstract}

Methods: Eighty-six patients undergoing thoracic surgery were randomized into two groups. Patientcontrolled epidural analgesia (PCEA) was used until chest tubes were removed. Patients received parecoxib (group P) or placebo (group C) intravenously just $0.5 \mathrm{~h}$ before the operation and every $12 \mathrm{~h}$ after operation for 3 days. The intensity of pain was measured by using a visual analogue scale (VAS) and recorded at 2, 4, 8, 24, 48, $72 \mathrm{~h}$ after operation. The valid number of PCA, the side effects and the overall satisfaction to analgesic therapy in $72 \mathrm{~h}$ were recorded. Venous blood samples were taken before operation, the $1^{\text {st }}$ and $3^{\text {rd }}$ day after operation for plasma cortisol, adrenocorticotropic hormone (ACTH), interleukin-6 and tumor necrosis factor- $\alpha$ level. The occurrence of residual pain was recorded using telephone questionnaire 2 and 12 months after surgery.

Results: Postoperative pain scores at rest and on coughing were significantly lower with the less valid count of PCA and greater patient satisfaction in group $\mathrm{P}(\mathrm{P}<0.01)$. Adverse effect and the days fit for discharge were comparable between two groups. The cortisol levels in placebo group were higher than parecoxib group at T2. The level of ACTH both decreased in two groups after operation but it was significantly lower in group $\mathrm{P}$ than that in group C. There were no changes in plasma IL-6 and TNF- $\alpha$ levels before and after analgesia at $\mathrm{T}_{1}$ and $\mathrm{T}_{2}(\mathrm{P}>0.05)$. The occurrence of residual pain were $25 \%$ and $51.2 \%$ separately in group $\mathrm{P}$ and $\mathrm{C}$ 3 months postoperatively $(\mathrm{P}<0.05)$.

Conclusions: Intravenous parecoxib in multimodal analgesia improves postoperative analgesia provided by TEA, relieves stress response after thoracotomy, and may restrain the development of chronic pain.

Keywords: Epidural analgesia; postoperative analgesia; parecoxib; thoracotomy

Submitted Nov 27, 2015. Accepted for publication Feb 14, 2016.

doi: $10.21037 /$ jtd.2016.03.45

View this article at: http://dx.doi.org/10.21037/jtd.2016.03.45

\section{Introduction}

Thoracotomy is considered as one of the most severe acute postoperative painful surgeries. Pain can be severe and come from numerous sources, including the skin incision and deeper tissue injuries, thoracostomy tubes, costovertebral joint disruption, and even fractures of the sternum or ribs (1). Although video-assisted thorascopic surgery (VATS) has been supported by studies reporting reduced incisional pain in the acute period when compared 
to thoracotomy (2), patients undergoing thoracotomy and VATS alike continue to experience chronic pain at an alarmingly high incidence $(3,4)$.

Uncontrolled acute perioperative pain and related surgical stress responses are highly associated with poor outcomes after thoracotomy and predict the development of chronic pain $(3,5)$. To decrease the incidence of complication after thoracic surgery, a multimodal analgesic strategy that includes regional anesthesia is considered to be a gold standard for patients undergoing thoracotomy and VATS $(6,7)$.

Nonsteroidal anti-inflammatory drugs (NSAIDs) reduce the production of prostaglandin by cyclooxygenase (COX) inhibition and are considered to be a good choice as adjuncts throughout the perioperative period (8). Their main advantage over opioids is effective pain relief without respiratory depression. Due to side effects of non-subtype selective $\mathrm{COX}$ inhibitor on gastrointestinal bleeding, coagulopathy, acute kidney injury and hypoperfusion, selected COX-2 inhibitor are more popular as adjuncts to TEA in acute thoracic pain management. Since its potential value as a treatment for acute perioperative pain is incompletely understood, we conducted this study to evaluate TEA sparing effect of parecoxib, a selected COX2 inhibitor, and its safety on perioperatively administer in general thoracic surgery.

\section{Methods}

\section{General design}

This was a single center, double-blind and prospective study on the multimodal analgesia of general thoracic surgery. After institutional approval and written informed consent were obtained, 86 patients under lobectomy from February to October 2014 in Zhongshan Hospital Fudan University were enrolled and randomized into two groups: placebo group (group $C, n=43$ ) or parecoxib group (group $P, n=43$ ).

Preoperative inclusion criteria were age between 18-75 years and ASA I-II. Exclusive criteria were coronary atery disease, peripheral vascular diseases, uncontrolled hypertension, increased concentration of liver enzyme (aspartate aminotransferase or alanine aminotransferase $>40 \mathrm{U} / \mathrm{L}$ ), creatinine level of greater than $133 \mathrm{mmol} / \mathrm{L}$, active peptic ulcer or peptic bleeding history, history of NSAIDs or sulfonamides allergy, asthma, chronic pain and long term treatment with analgesics (e.g., NSAIDS, opioids) or steroids, paresthesia of peripheral neuropathy, pregnant or breastfeeding and contraindications of epidural puncture. Postoperative exclusive criteria were operating time $>4$ hrs and failure epidural analgesia.

\section{Protocol}

Epidural catheters were placed at T7-8 intervertebral spaces before anesthesia induction. The effect of epidural analgesia was confirmed before induction. Anesthesia induction was performed with fentanyl $(2 \mu \mathrm{g} / \mathrm{kg})$, remifentanil $(0.15 \mu \mathrm{g} / \mathrm{kg} / \mathrm{min}$ ), propofol (TCI Ce $=2.5 \mu \mathrm{g} / \mathrm{mL}$ ) and rocuronium $(0.6 \mathrm{mg} / \mathrm{kg})$. Double lumen tracheal tube was intubated after induction and fiberoptic bronchoscopy was used to confirm its position. Anesthesia was maintained with desflurane (0.6-0.8 MAC), propofol (Ce $=1 \mu \mathrm{g} / \mathrm{mL})$, fentanyl and rocuronium. $0.1875 \%$ ropivacaine was used epidurally after induction with $15 \mathrm{~mL}$ as loading dose and $5 \mathrm{~mL}$ at $1 \mathrm{hr}$-interval. Epidural morphine $(2 \mathrm{mg})$ and intravenous tropisetron mesylate were used $30 \mathrm{~min}$ before the end of operation. All the patients were extubated within $10 \mathrm{~min}$ after operation and transferred to PACU.

\section{Administration of multimodal analgesia}

Intravenous administration of the study drug $(40 \mathrm{mg}$ of parecoxib or placebo) began 30 minutes before intubation of the trachea following a baseline pain assessment. The study drug was then administered intravenously every 12 hours and was continued for 60 hours. After admitted to PACU, patient-controlled epidural analgesia started with the formulation of $0.1 \%$ bupivacaine, $2 \mu \mathrm{g} / \mathrm{mL}$ fentanyle and $12 \mu \mathrm{g} / \mathrm{mL}$ morphine. Infusion rate was $3-4 \mathrm{~mL} / \mathrm{h}$, bolus volume was $4-5 \mathrm{~mL}$ and lock time was $10 \mathrm{~min}$. If VAS $>3$ after bolus epidural analgesics, supplemental pain medication consisting of $5 \mathrm{mg}$ oxycodone was used orally.

\section{Evaluation of analgesic efficacy}

The primary measure for comparing efficacy of multimodal analgesia versus epidural analgesia only was the amount of demanding dose of epidural analgesia and supple-mental pain medication. To further assess the quality of pain control, the patients' VAS scores with 0 corresponding to no pain and 10 corresponding to the worst pain imaginable were recorded at 2, 4, 8, 24, 48 and $72 \mathrm{hrs}$ both in rest and on coughing. Additional assessments included intensity and characteristic of residual pain related to the surgical site (one month after dischargement). To evaluate the distress 


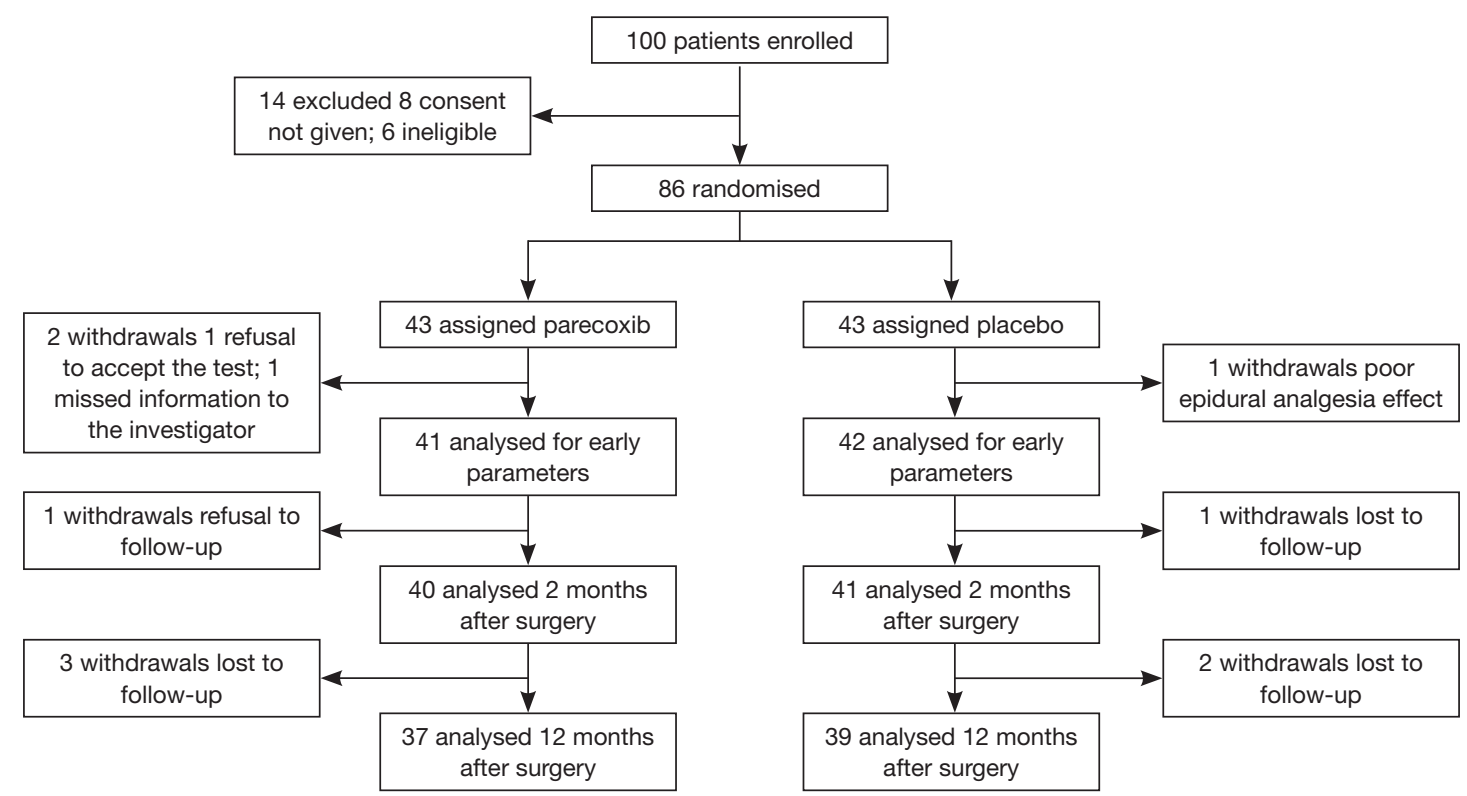

Figure 1 Diagram showing the flow of the patients enrolled in the study. Eighty-six patients were included before surgery and 83 ones analysed for early parameters.

of acute postoperative pain, blood samples were drawn respectively before anesthesia induction (T0), postoperative day 1 (T1) and postoperative day 2 (T2) for adrenocorticotropic hormone (ACTH), cortisol IL6 and TNF- $\alpha$.

\section{Safety assessment}

Safety was assessed on the basis of the occurrence of clinical adverse events (AEs) including surgical AEs and analgesia associated events. Analgesia related AEs referred to hypotension (a decrease in arterial pressure below $90 / 60 \mathrm{mmHg}$ ), postoperative nausea and vomiting (VAS 0, no symptom; 1-3, mild; 4-6, moderate; 7-10, severe), dizziness and pruritus.

\section{Statistical analysis}

The quantitative data were expressed as mean and standard deviation if normally distributed and as quartiles and range otherwise. The categorical data were expressed as number of cases and percentage of the total. Student's $t$-test, ANOVA for repeated measures, or the MannWhitney test was used for quantitative data. Fischer exact test was used to compare qualitative data. A value of $\mathrm{P}<0.05$ was considered significant. The results of a pilot study in our institution indicated that 36 subjects per group allowed detection of a $20 \mathrm{~mm}$ reduction in VAS pain score 3 months after thoracotomy with $\alpha=0.05$ and a $90 \%$ power.

\section{Results}

Eighty-six patients were included before surgery and 83 ones completed this study (see flow chart in Figure 1). Demographic and operation related data of these patients are presented in Table 1.

Postoperative PCEA data are shown in Table 2. Compared to group C, patient's demand count and invalid delivery count for bolus dose was significantly decreased in group $\mathrm{P}$ (patient's demand count: $5.3 \pm 3.9$ vs. $15.3 \pm 5.5$; invalid delivery count: $1.3 \pm 1.6$ vs. $3.5 \pm 3.4)(\mathrm{P}<0.01)$. The total PCEA volume delivered in group $\mathrm{C}$ was higher than group $\mathrm{P}(16.1 \pm 12.6$ vs. $47.3 \pm 17.3 \mathrm{~mL})(\mathrm{P}<0.01)$.

During PCEA therapy, VAS scores especially on coughing were significantly lower in group $\mathrm{P}$ than group $\mathrm{C}$ (Figure 2). Nine patients in group $\mathrm{C}$ took oxycodone as rescue therapy compared to 2 in group $\mathrm{P}$. The total amount of oxycodone used in both group was same (Table 3) $(\mathrm{P}>0.05)$.

Laboratory tests for plasma ACTH, cortisol, IL6 and TNF- $\alpha$ (Tables 4,5 and Figures 3,4). There was no difference on baseline of ACTH, cortisol IL6 and TNF- $\alpha$ between group $\mathrm{C}$ and group P. ACTH was significantly reduced 
Table 1 Demographic and surgical characteristics of the patients at the time of randomisation

\begin{tabular}{|c|c|c|c|}
\hline Characteristics & Group P $(n=41)(\%)$ & Group C (n=42) (\%) & $P$ value \\
\hline Sex ratio $(M / F)$ & $19 / 22(46.3 / 53.6)$ & $24 / 18(57.1 / 42.9)$ & 0.325 \\
\hline Age (yr) & $37-76$ & $22-72$ & \\
\hline $40-50$ & $7(17.0)$ & $6(14.3)$ & \\
\hline $50-60$ & $13(31.7)$ & $11(26.2)$ & \\
\hline$>70$ & $5(12.2)$ & $6(14.3)$ & \\
\hline Means \pm SD & $55.9 \pm 10.5$ & $57.1 \pm 12.0$ & 0.625 \\
\hline Body weight (kg) & $66.0 \pm 9.7$ & $66.6 \pm 6.9$ & 0.769 \\
\hline Height $(\mathrm{cm})$ & $164.9 \pm 6.2$ & $166.8 \pm 7.0$ & 0.199 \\
\hline ASA (I/II) & 18/23 (43.9/56.1) & 20/22 (47.6/52.4) & 0.734 \\
\hline Lobectomy & $30(73.2)$ & $32(76.2)$ & \\
\hline Wedge resection & $8(19.5)$ & $10(23.8)$ & \\
\hline Surgery time (min) & $126.9 \pm 40.5$ & $127.0 \pm 36.5$ & 0.982 \\
\hline Rib spreader/trocar time (min) & $101.2 \pm 38.3$ & $96.9 \pm 33.1$ & 0.578 \\
\hline
\end{tabular}

ASA, American Society of Anesthesiologists; VATS, video-assisted thorascopic surgery; Numerical data are expressed as means \pm SD.

Table 2 PCEA within 72 hours postoperatively

\begin{tabular}{lcccc}
\hline Groups & Patient demand & Valid demand & Invalid demand & Total volume of PCEA $(\mathrm{mL})$ \\
\hline Group P $(\mathrm{n}=41)$ & $5.3 \pm 3.9$ & $4.0 \pm 3.1$ & $1.3 \pm 1.0$ & $16.1 \pm 10.6$ \\
Group C $(\mathrm{n}=42)$ & $15.3 \pm 5.5$ & $11.8 \pm 4.3$ & $3.5 \pm 2.2$ & $47.3 \pm 17.3$ \\
\hline
\end{tabular}

PCEA, patient-controlled epidural analgesia.
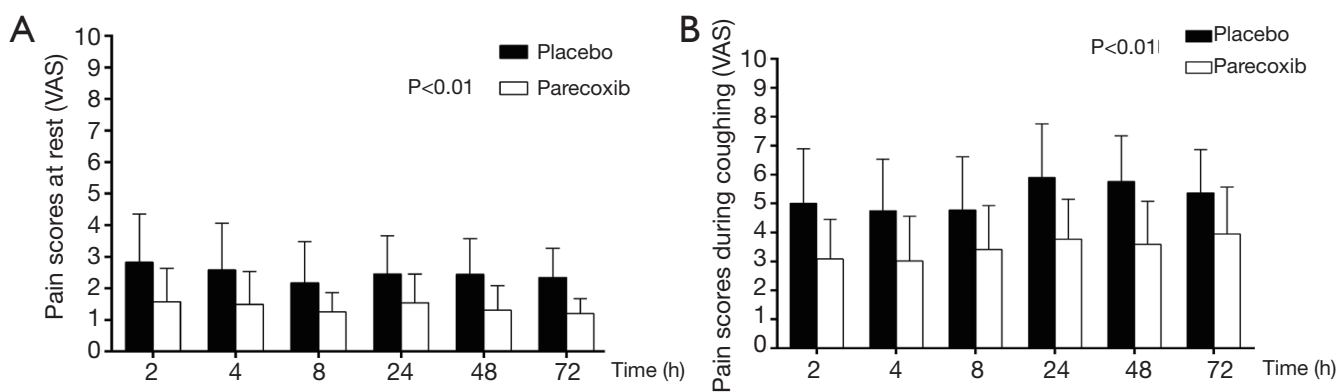

Figure 2 Postoperative VAS score at rest and on coughing. VAS scores were significantly lower in group P than group C in 72 h after surgery. VAS, visual analogue scale. 
Table 3 Quality of the postoperative analgesia

\begin{tabular}{lccc}
\hline Analgesic effect & Group P $(\mathrm{n}=41)$ & Group C $(\mathrm{n}=42)$ & $\mathrm{P}$ value \\
\hline Cases of rescue drug use $(\%)$ & $2(4.9)$ & $9(21.4)$ & 0.026 \\
Rescue drug usage $(\mathrm{mg})$ & $10 \pm 5$ & $17.8 \pm 9.5$ & 0.331 \\
VAS of analgesia satisfaction & $7.89 \pm 1.39$ & $6.16 \pm 1.49$ & $<0.01$ \\
\hline
\end{tabular}

VAS, visual analogue scale; Numerical data are expressed as means \pm SD.

Table 4 Plasma concentration of ACTH and cortisol

\begin{tabular}{lcccc}
\hline Stress hormone & Groups & Day 0 (T0) & Day 1 (T1) & Day 3 (T2) \\
\hline ACTH $(\mathrm{pg} / \mathrm{mL})$ & Group P $(\mathrm{n}=41)$ & $56.5 \pm 25.0$ & $27.1 \pm 15.3^{\text {*,\# }}$ & $20.7 \pm 7.8^{\text {*,\# }}$ \\
& Group C $(\mathrm{n}=42)$ & $60.3 \pm 20.0$ & $39.8 \pm 19.9^{*}$ & $30.4 \pm 9.3^{*}$ \\
Cortisol $(\mathrm{nmol} / \mathrm{L})$ & Group P $(\mathrm{n}=41)$ & $502.1 \pm 160.1$ & $503.2 \pm 183.9^{\#}$ & $380.6 \pm 100.4^{*, \#}$ \\
& Group C $(\mathrm{n}=42)$ & $502.5 \pm 125.4$ & $744.5 \pm 225.5^{*}$ & $545.3 \pm 189.3$ \\
\hline
\end{tabular}

*, compared to T0, $\mathrm{P}<0.05$; ", compared to group $\mathrm{C}, \mathrm{P}<0.05$. ACTH, adrenocorticotropic hormone.

Table 5 Plasma concentration of TNF- $\alpha$ and IL6

\begin{tabular}{lllll}
\hline Cytokine & \multicolumn{1}{c}{ Groups } & Day 0 (T0) & Day 1 (T1) & Day 3 (T2) \\
\hline TNF- $\alpha(\mathrm{ng} / \mathrm{mL})$ & Group P $(\mathrm{n}=41)$ & $1.50 \pm 0.17$ & $1.49 \pm 0.20$ & $1.45 \pm 0.21$ \\
& Group C $(\mathrm{n}=42)$ & $1.47 \pm 0.18$ & $1.46 \pm 0.14$ & $30 \pm 0.21$ \\
IL-6 $(\mathrm{pg} / \mathrm{mL})$ & Group P $(\mathrm{n}=41)$ & $36.6 \pm 5.5$ & $36.4 \pm 5.0$ & $38.6 \pm 3.7$ \\
& Group C $(\mathrm{n}=42)$ & $35.4 \pm 6.8$ & $35.3 \pm 7.2$ & $38.5 \pm 7.2$ \\
\hline
\end{tabular}

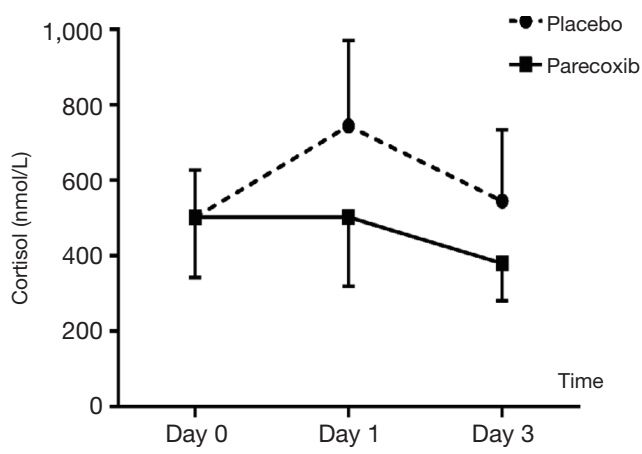

Figure 3 Plasma concentration changes of cortisol. The cortisol concentration was significantly lower in group $\mathrm{P}$ than group $\mathrm{C}$ on day 1 and day 3 after operation.

postoperatively in both groups while the level was further lower in group P. Postoperative cortisol was significantly lower in group P than group C. As to IL6 and TNF- $\alpha$, there were no difference between two groups or among three time points (T0, T1 and $\mathrm{T} 2)$.

Table 6 shows the residual pain related to incision site 2

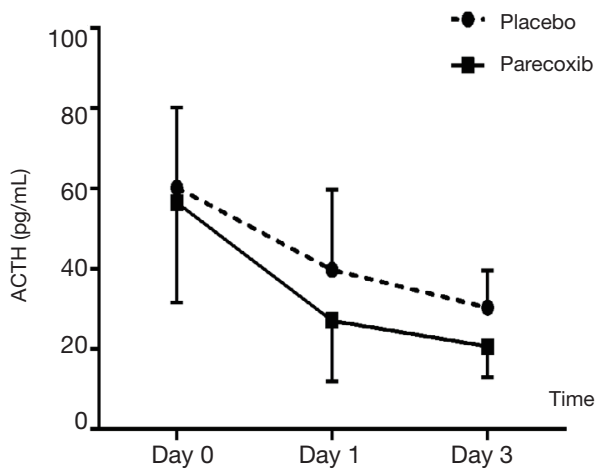

Figure 4 Plasma concentration changes of ACTH. In two groups, plasma concentration of ACTH both significantly reduced postoperatively, especially in patients treated with parecoxib. ACTH, adrenocorticotropic hormone.

and 12 months after surgery. Eighty-one patients completed telephone questionnaire at the first follow-up and 76 patients finally completed the study. Group P had lower incidence and intensity of incision site related pain.

All the AEs were listed in Table 7. There were no differences between the groups with respect to side effects. 
Table 6 Chronic pain related to incision site after surgery

\begin{tabular}{|c|c|c|c|c|c|c|}
\hline \multirow{2}{*}{ Occurrence of chronic pain } & \multicolumn{3}{|c|}{3 months } & \multicolumn{3}{|c|}{12 months } \\
\hline & Group C & Group P & $P$ value & Group C & Group P & $P$ value \\
\hline Incision related pain (\%) & $20 / 41(48.9)$ & $9 / 40(22.5)$ & 0.014 & 14/39 (35.9) & $5 / 37(13.5)$ & 0.024 \\
\hline
\end{tabular}

Table 7 Recorded adverse event within 72 hrs after surgery

\begin{tabular}{|c|c|c|c|}
\hline Type of event & Group P $(n=41)(\%)$ & Group C (n=42) (\%) & $P$ value \\
\hline \multicolumn{4}{|l|}{ Analgesia related } \\
\hline Hypotension & $5(12.2)$ & $3(7.1)$ & 0.683 \\
\hline Dizziness & $3(7.3)$ & $2(4.8)$ & 0.978 \\
\hline Pruritus & $0(0)$ & $1(2.4)$ & 1.000 \\
\hline Nausea and vomitting & $6(14.6)$ & $7(16.7)$ & 0.799 \\
\hline Mild & $3(7.3)$ & $2(4.7)$ & \\
\hline Moderate & $2(4.9)$ & $4(9.5)$ & \\
\hline Severe & $1(2.4)$ & $1(2.4)$ & \\
\hline \multicolumn{4}{|l|}{ Surgery related } \\
\hline Arrhythmia & $1(2.4)$ & $3(7.1)$ & 0.626 \\
\hline High fever $\left(T>38^{\circ} \mathrm{C}\right)$ & $1(2.4)$ & $4(9.5)$ & 0.371 \\
\hline Liver dysfunction & $5(12.2)$ & $5(11.9)$ & 1.000 \\
\hline Renal dysfunction & $5(12.2)$ & $1(2.4)$ & 0.193 \\
\hline Abnormal coagulation & $1(2.4)$ & $1(2.4)$ & 1.000 \\
\hline Others & $2(4.9)$ & $0(0)$ & 0.464 \\
\hline
\end{tabular}

\section{Discussion}

Our study demonstrated that perioperative intravenous parecoxib had great TEA sparing effect on acute postoperative pain and decreased incidence of surgical site related pain 3 and 12 months later. At the same time surgery related stress was also suppressed and immune injury alleviated. The incidence of complication whether surgery related or analgesia related had no difference between two groups which indicated the safe use of parecoxib in acute postoperative pain management.

It has been widely accepted that epidural analgesia effectively blunt the stress response to surgery and provide superior pain relief compared to systemic opioids for patients undergoing thoracic surgery (9). Thoracic epidural analgeisa (TEA) by PCEA model have been extensively studied and regarded as the standard for analgesia in patients undergoing thoracic surgery (10).

It was in the late of 1980s, combination of epidural and intravenous postoperative analgesia came into existence.
Besides its total relief of pain, no significant effect on surgical stress and pulmonary function was observed. In view of this, Kehlet and Dahl (6) bring forward the concept of multimodal or balanced analgesia to achieve sufficient analgesia due to additive or synergistic effects between different analgesics with concomitant reduction of side effects which was a result of lower doses and differences in side-effect profiles of analgesics.

Pavy et al. (11) first reported opiods sparing effect of NSAIDs (indomethacin) in thoracotomy. Numerous NSAIDs are available with varying selectivity for the two types of COX (I and II). According to two studies from Connail M (12), COX-2 selective NSAIDs reduced synthesis of spinal PG and played a key role in post-thoracotomy analgesia. So we selected parecoxib (an intravenous selective COX-2) as an adjuvant of PCEA to evaluate its effect and safety on multimodal analgesia.

Most of previous study of parecoxib were on the orthopedic surgery and showed that intravenous parecoxib did reduce the pain score postoperatively $(13,14)$. Argiriadou 
et al. (15) first carried out a study to find out the effect of parecoxib combined with thoracic paravertebral ropivacaine infusion in thoracotomy. Patients receiving parecoxib (40 $\mathrm{mg}$ intravenously used $20 \mathrm{~min}$ before extubation and 12 hrs after the procedure) had less pain at rest and during movement compared with those with placebo at 12, 24, and 48 hours after surgery. In accordance with their study, we found out that perioperative use of parecoxib significantly reduced the pain score and additional PCEA and there was no cardiovascular AE recorded associated with its use. Our study also included the concept of preemptive use of parecoxib. Preemptive analgesia is a concept that a pain therapy is more effective if given before the surgical incision and noxious stimulus. It is thought to decrease the incidence of hyperalgesia and allodynia by decreasing the altered central sensory processing (16). The first dosage of parecoxib in this study was used $30 \mathrm{~min}$ before surgery to maximize its adjuvant effect on pain management.

Post-thoracotomy pain lasting more than two months is identified as chronic. This complex is also known as postthoracotomy pain syndrome (PTPS) (17). The reported incidence is quite high, ranging from $30 \%$ to $80 \%$ by varied studies but without difference between VATS and thoracotomy $(3,18)$. In this study we questionnaire the patients by telephone three months after procedure and found that parecoxib significantly reduced the incision related pain. Results from longer follow-up (twelve months) time further proved the conclusion of parecoxib on PTPS.

Enhanced Recovery After Surgery (ERAS) attracts more and more attention of clinicians including surgeons, anesthesiologist and ICU faculties who are intent on minimizing postoperative morbidity, speeding up recovery and reducing hospital costs. Appropriate systemic inflammatory response is essential for many beneficial aspects of wound healing while an exaggerated inflammatory response has been associated with adverse perioperative outcomes. Patients with higher proinflammatory responses were shown to be prone to a greater incidence of postoperative complications (19). Hypothalamic-pituitary-adrenal axis was involved in the response of nociceptive stimuli. Several studies showed clearly that pain itself could trigger the change of endocrine and inflammatory response $(20,21)$. Epidural anesthetics could decrease the level of stress though hormonal (cortisol) and inflammatory response attenuation. But no data was available for NSAIDs. From this study we found that plasma concentration of ACTH significantly reduced postoperatively especially after parecoxib. As to cortisol, parecoxib decreased postoperative plasma level especially compared to group $\mathrm{C}$ in which the concentration significantly increased on day 1 after operation. This dissociation of ACTH and cortisol level has been reported in some surgical situations (22). Cho et al. (23) reported $30 \%$ of patients exhibited a sustained increased cortisol level while the concentration of ACTH returned to preoperative levels in the early postoperative stage of gastrectomy. This dissociation was relative to more postoperative complications.

Early Inflammatory cytokines IL-6 and TNF- $\alpha$ did not significantly increased in both group and showed no difference between two groups in our study. This might due to the effect of epidural analgesia on stress depression (24).

In conclusion this is the first report of multimodal analgesia of thoracic surgery involving the PCEA sparing effect and lowering the surgical stress of parecoxib. More significant is that our study indicated multimodal analgesia including parecoxib (an COX-2 inhibitor) might reduce the incidence of PTPS which had a prevalence of $24.3 \%$ with reported impaired the performance of activities of daily living, anxiety or depression and sleeping disturbance two months after surgery.

Unfortunately this study had some deficits: first, we did not evaluated pulmonary function before and after operation; secondly, we did not compare the effect of intravenous opioids and COX-2. More cases should be included to evaluation the safety of perioperative COX2 inhibitor administration and its long-term effect on recovery after thoracic surgery in future.

\section{Acknowledgements}

This work was supported by the research foundation from The 2nd Oriental Congress of Anesthesiology and Perioperative Medicine for the research on optimizing the intraoperative ventilation strategy in minimal invasive thoracic surgery.

\section{Footnote}

Conflicts of Interest: The authors have no conflicts of interests to declare.

\section{References}

1. Ochroch EA, Gottschalk A. Impact of acute pain and its 
management for thoracic surgical patients. Thorac Surg Clin 2005;15:105-21.

2. Furrer M, Rechsteiner R, Eigenmann V, et al. Thoracotomy and thoracoscopy: postoperative pulmonary function, pain and chest wall complaints. Eur J Cardiothorac Surg 1997;12:82-7.

3. Wildgaard K, Ravn J, Kehlet H. Chronic postthoracotomy pain: a critical review of pathogenic mechanisms and strategies for prevention. Eur J Cardiothorac Surg 2009;36:170-80.

4. Gottschalk A, Cohen SP, Yang S, et al. Preventing and treating pain after thoracic surgery. Anesthesiology 2006;104:594-600.

5. Kehlet H, Jensen TS, Woolf CJ. Persistent postsurgical pain: risk factors and prevention. Lancet 2006;367:1618-25.

6. Kehlet H, Dahl JB. The value of "multimodal" or "balanced analgesia" in postoperative pain treatment. Anesth Analg 1993;77:1048-56.

7. White PF, Kehlet H. Improving postoperative pain management: what are the unresolved issues? Anesthesiology 2010;112:220-5.

8. Kaye AD, Baluch A, Kaye AJ, et al. Pharmacology of cyclooxygenase-2 inhibitors and preemptive analgesia in acute pain management. Curr Opin Anaesthesiol 2008;21:439-45.

9. Thompson JS. The role of epidural analgesia and anesthesia in surgical outcomes. Adv Surg 2002;36:297-307.

10. Von Dossow V, Welte M, Zaune U, et al. Thoracic epidural anesthesia combined with general anesthesia: the preferred anesthetic technique for thoracic surgery. Anesth Analg 2001;92:848-54.

11. Pavy T, Medley C, Murphy DF. Effect of indomethacin on pain relief after thoracotomy. Br J Anaesth 1990;65:624-7.

12. Maxwell C, Nicoara A. New developments in the treatment of acute pain after thoracic surgery. Curr Opin Anaesthesiol 2014;27:6-11.

13. Malan TP Jr, Marsh G, Hakki SI, et al. Parecoxib sodium, a parenteral cyclooxygenase 2 selective inhibitor, improves morphine analgesia and is opioid-sparing following total

Cite this article as: Ling XM, Fang F, Zhang XG, Ding M, Liu QA, Cang J. Effect of parecoxib combined with thoracic epidural analgesia on pain after thoracotomy. J Thorac Dis 2016;8(5):880-887. doi: 10.21037/jtd.2016.03.45 hip arthroplasty. Anesthesiology 2003;98:950-6.

14. Kranke P, Morin AM, Roewer N, et al. Patients' global evaluation of analgesia and safety of injected parecoxib for postoperative pain: a quantitative systematic review. Anesth Analg 2004;99:797-806, table of contents.

15. Argiriadou H, Papagiannopoulou P, Foroulis CN, et al. Intraoperative infusion of $\mathrm{S}(+)$-ketamine enhances postthoracotomy pain control compared with perioperative parecoxib when used in conjunction with thoracic paravertebral ropivacaine infusion. J Cardiothorac Vasc Anesth 2011;25:455-61.

16. Erturk E, Aydogdu Kaya F, Kutanis D, et al. The effectiveness of preemptive thoracic epidural analgesia in thoracic surgery. Biomed Res Int 2014;2014:673682.

17. Merskey H, Bogduk H. Classification of chronic pain. In: Merskey H, Bogduk H, editors. Descriptions of chronic pain syndromes and definitions of pain terms. Second Edition, Seattle, WA: ISAP. Press, 1994:143-4.

18. Bottiger BA, Esper SA, Stafford-Smith M. Pain management strategies for thoracotomy and thoracic pain syndromes. Semin Cardiothorac Vasc Anesth 2014;18:45-56.

19. Carli F. Physiologic considerations of Enhanced Recovery After Surgery (ERAS) programs: implications of the stress response. Can J Anaesth 2015;62:110-9.

20. Epstein J, Breslow MJ. The stress response of critical illness. Crit Care Clin 1999;15:17-33, v.

21. Kim PK, Deutschman CS. Inflammatory responses and mediators. Surg Clin North Am 2000;80:885-94.

22. Bornstein SR, Engeland WC, Ehrhart-Bornstein M, et al. Dissociation of ACTH and glucocorticoids. Trends Endocrinol Metab 2008;19:175-80.

23. Cho YM, Kim SY, Cho BY, et al. Dissociation between plasma adrenocorticotropin and serum cortisol level during the early postoperative period after gastrectomy. Horm Res 2000;53:246-50.

24. Doan LV, Augustus J, Androphy R, et al. Mitigating the impact of acute and chronic post-thoracotomy pain. J Cardiothorac Vasc Anesth 2014;28:1048-56. 